\section{Archives of Allergy \\ and Immunology}

Int Arch Allergy Immunol 2021;182:1026-1035

DOI: $10.1159 / 000516417$
Received: February 22, 2021

Accepted: April 7, 2021

Published online: June 3, 2021

\title{
MP-AzeFlu in Moderate-to-Severe Allergic Rhinitis: A Literature Review
}

\author{
Ludger Klimek $^{a}$ William E. Berger ${ }^{b}$ Jean Bousquet ${ }^{c, d}$ Paul K. Keith $^{e}$ \\ Peter Smith $^{f}$ Dirceu Sole ${ }^{g}$ Glenis Scadding ${ }^{h}$ Hans Christian Kuhl ${ }^{i}$ \\ Duc Tung Nguyen ${ }^{i}$ Ferdinand Kopietz ${ }^{i}$ Arkady Koltun ${ }^{j}$
}

${ }^{a}$ Center for Rhinology and Allergology, Wiesbaden, Germany; ${ }^{b}$ Allergy and Asthma Associates of Southern California, Mission Viejo, CA, USA; ${ }^{C} \mathrm{CHU}$ Montpellier, Montpellier, France; ${ }^{\mathrm{d}}$ Charité - Universitätsmedizin Berlin, Humboldt-Universität zu Berlin, Berlin, Germany; ${ }^{e}$ Division of Allergy and Clinical Immunology, Department of Medicine, McMaster University, Hamilton, ON, Canada; ${ }^{f}$ Queensland Allergy Services, Southport, QLD, Australia; 9Division of Allergy, Department of Pediatrics, Clinical Immunology and Rheumatology, Federal University of São Paulo/UNIFESP, São Paulo, Brazil; hroyal National Throat Nose and Ear Hospital, London, United Kingdom; 'MEDA Pharma GmbH \& Co. KG (A Mylan Company), Bad Homburg, Germany; 'Mylan, Inc., Canonsburg, PA, USA

\section{Keywords}

Allergic rhinitis - Azelastine · Fluticasone propionate . MP-AzeFlu · Nasal symptoms · Ocular symptoms · Total nasal symptom score

\begin{abstract}
Allergic rhinitis (AR) is prevalent, and many patients present with moderate-to-severe symptomatic disease. The majority of patients are not satisfied with their AR treatment, despite the use of concurrent medications. These gaps underscore the need for treatment with more effective options for moderate-to-severe AR. The authors' objective was to review systematically the efficacy and safety of MP-AzeFlu for the treatment of AR. The primary outcomes studied were nasal, ocular, and total symptoms. Other outcomes included time to onset and of AR control, quality of life, and safety. Searches of PubMed and Cochrane databases were conducted on May 14, 2020, with no date restrictions, to identify publications reporting data
\end{abstract}

karger@karger.com www.karger.com/iaa

Karger $\stackrel{\text { ' }}{5}$
(C) 2021 The Author(s)

Published by S. Karger AG, Basel

This is an Open Access article licensed under the Creative Common Attribution-NonCommercial-4.0 International License (CC BY-NC) (http://www.karger.com/Services/OpenAccessLicense), applicable to the online version of the article only. Usage and distribution for commercial purposes requires written permission. on MP-AzeFlu. Clinical studies of any phase were included. Studies were excluded if they were not in English, were review articles, did not discuss the safety and efficacy of MPAzeFlu for AR symptoms. Treatment of AR with MP-AzeFlu results in effective, sustained relief of nasal and ocular symptoms, and faster onset and time to control compared with intranasal azelastine or fluticasone propionate. Longterm use of MP-AzeFlu was safe, with benefits in children, adults, and adults aged $\geq 65$ years. Other treatment options, including fluticasone propionate and azelastine alone or the combination of intranasal corticosteroids and oral antihistamine, do not provide the same level of efficacy as MP-AzeFlu in terms of rapid and sustained relief of the entire AR symptom complex. Furthermore, MP-AzeFlu significantly improves patient quality of life. MP-AzeFlu is a currently available combination that may satisfy all these patient needs and expectations.

(c) 2021 The Author(s).

Published by S. Karger AG, Basel

Edited by: D.Y. Wang, Singapore. 


\section{Introduction}

\section{Allergic Rhinitis}

Allergic rhinitis (AR) is prevalent, and many patients present with moderate-to-severe symptomatic disease [1]. Moderate-to-severe AR can be classified based on the presence of at least one of the following symptoms: sleep disturbance; impairment of daily activities, leisure, and/ or sport; impairment of school or work; and troublesome symptoms [1]. Patients with AR often have comorbidities such as rhinosinusitis, sleep disturbance, otitis media, and asthma [1-3]. The symptom complex of AR, which includes nasal and ocular symptoms, can have a profound impact on quality of life, work productivity, and school performance $[1,4]$.

Therapy options for AR depend on many factors, including symptom severity and control. Pharmacologic approaches should strive for immediate and sustained symptom relief. Among patients receiving treatment, $43 \%$ expect allergy symptom suppression, $12 \%$ expect to be cured, and $20 \%$ expect both symptom suppression and allergy cure [5]. Patients are willing to pay more to achieve more complete and faster AR symptom relief [6].

Most patients with moderate-to-severe AR are not satisfied with their current treatment, despite the use of concurrent medications [7]. Many patients use on-demand treatment when symptoms are suboptimally controlled, which may lead to daily medication changes [8]. Oral antihistamines are most often used, although they are not the most effective treatment [9]. Poor medication choices lead to undermanaged disease and may contribute to morbidity [10].

AR affects up to $40 \%$ of children, whose parents may be unaware of symptom presence or severity ("nasal neglect") [1]. These issues are compounded by adverse effects of medications used, including sedating antihistamines or medications, which are only licensed for adults [11]. Effective treatments are needed for patients of all ages with AR.

\section{MP-AzeFlu}

MP-AzeFlu is a fixed-dose combination product containing an $\mathrm{H}_{1}$-receptor antagonist (azelastine hydrochloride; AZE) and a corticosteroid (fluticasone propionate; FP) that is administered intranasally [12]. AZE and FP have demonstrated synergistic effects regarding improvement of AR symptoms, with inhibition of the synthesis or release of chemical mediators involved in early- and latestage allergic reactions (AZE) as well as potent anti-inflammatory activities (FP). The nasal spray delivers 137 mcg of AZE and $50 \mathrm{mcg}$ of FP $(137 / 50 \mathrm{mcg})$ in each 0.137 $\mathrm{mL}$ spray. The recommended MP-AzeFlu dosage is 1 spray/nostril twice daily. The indicated age for MP-AzeFlu varies by country. MP-AzeFlu is recommended by the US Joint Task Force on Practice Parameters as a first-line option for patients with moderate-to-severe AR, and the Allergic Rhinitis and its Impact on Asthma (ARIA) guidelines recommend MP-AzeFlu for all patients with AR, independent of disease type or severity $[8,13,14]$.

MP-AzeFlu allows for better deposition compared with sequential administration of its components and improved absorption of FP versus the generic formulation, likely because of the decreased volume required for MP-AzeFlu dosing [15]. MP-AzeFlu also has a larger spray volume, lower viscosity, and a finer droplet size distribution profile, resulting in a larger spray pattern diameter, superior dispersion, and larger total area versus FP-Boehringer-Ingelheim [16]. Finally, the pH of MP-AzeFlu is 6.0, which is more neutral than other formulations [11]. These biopharmaceutical characteristics may contribute to improved clinical efficacy of MP-AzeFlu via more active ingredients remaining at the targeted tissue, which leads to enhanced symptom reduction. This review was designed to systematically evaluate the current literature to determine the safety and efficacy of MP-AzeFlu for the treatment of AR.

\section{Materials and Methods}

Searches of the PubMed and Cochrane databases were conducted on May 14, 2020, with no date restrictions, to identify publications reporting data on MP-AzeFlu. The primary outcomes studied were nasal symptoms, ocular symptoms, and total symptoms. Other outcomes studied included time to onset of effect, time to AR control, effects on disease-specific quality of life, and safety. In brief, the search strategy included the terms "MP-AzeFlu," "MP 29-02," "Dymista," "formulation of azelastine hydrochloride and fluticasone propionate," and "azelastine and fluticasone in a single spray." Research studies of any phase were included; in vitro studies were excluded. Studies were excluded if they were not published in English; if they were review articles or conference abstracts; or if they did not discuss data related to the safety and efficacy of MP-AzeFlu treatment for AR symptoms. Country-specific data from multicenter studies were excluded, except Kaulsay and colleagues [17], because additional endpoints related to nasal mucosal examination were assessed. Because a combination of clinical trials and real-world studies with various primary endpoints were included, a meta-analysis was not suitable.

A single reviewer conducted preliminary screening of all publications identified to assess titles and abstracts according to the eligibility criteria. Consensus to include screened studies was reached with a second reviewer. Any discrepancies were resolved by a third reviewer. After inclusion confirmation, a reviewer extracted relevant data and a second reviewer provided validation of the findings. 
Table 1. MP-AzeFlu studies [17-31]

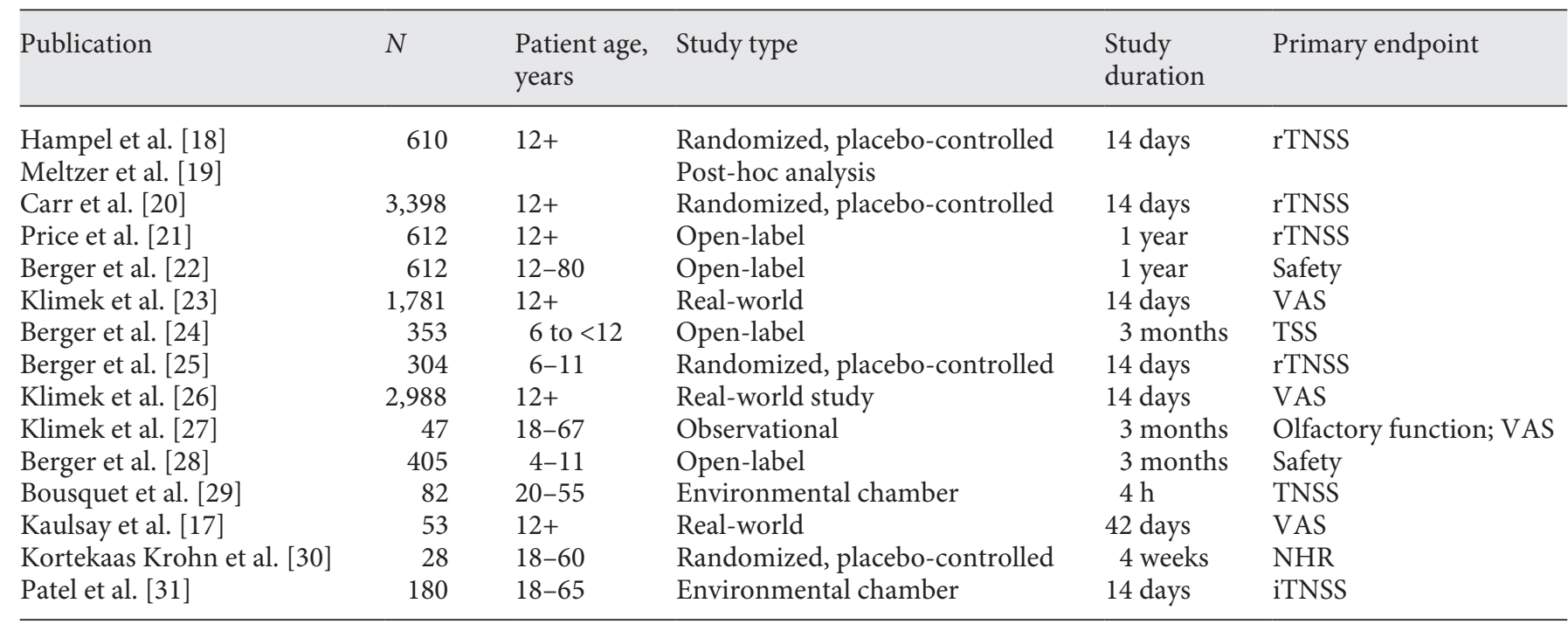

iTNSS, instantaneous total nasal symptom score; NHR, nasal hyperreactivity; TNSS, total nasal symptom score; rTNSS, reflective total nasal symptom score; TSS, total symptom score; VAS, visual analog scale.

\section{Results}

Of 103 publications screened (see online suppl. Table 1 at www.karger.com/doi/10.1159/000516417), 16 met the inclusion criteria and were evaluated. Excluded were 52 duplicate publications, 29 unrelated or review articles, and 6 articles publishing data from individual countries when a pooled analysis was available. Among these, studies described effects on nasal symptoms, ocular symptoms, time to AR control, quality of life, safety, and in special populations, as well as time to onset of effect, as described in the remainder of this review (Table 1).

\section{Nasal Symptoms: Efficacy and Time to Control}

The presence of nasal symptoms is associated with poor AR control and decreased health-related quality of life and can occur among patients using intranasal corticosteroids and/or oral or intranasal antihistamines [32]. Therefore, disease severity may be underestimated and inadequately treated, with many patients experiencing severe symptoms despite monotherapy use [8]. Real-world evidence supports the recommendation to use a more effective combination of intranasal $\mathrm{H}_{1}$-antihistamines with intranasal corticosteroids versus intranasal corticosteroids alone among patients with AR $[8,32]$.

MP-AzeFlu achieved a 44-64\% greater nasal symptom improvement versus its component comparators (FP and
AZE) in key pivotal trials [20]. In a pooled analysis of 3,398 patients aged $\geq 12$ years with moderate-to-severe seasonal AR (SAR) enrolled in 3 multicenter, randomized, doubleblind, placebo- and active-controlled, parallel-group trials, Carr and colleagues [20] investigated the efficacy of MPAzeFlu (MP29-02) versus AZE, FP, and placebo; all treatments were administered in the same device with the same vehicle formulation. Over the 14-days treatment period, MP-AzeFlu led to a significantly greater reduction of mean reflective total nasal symptom score versus FP, AZE, or placebo ( -5.7 vs. $-5.1,-4.4$, or -3.0 , respectively; all $p<0.001$ ). The superiority of MP-AzeFlu to FP and AZE was seen for all individual nasal symptoms. Among patients who experienced a clinically meaningful reduction in nasal symptoms ( $\geq 50 \%$ reduction in reflective total nasal symptom score, as defined in this study), those treated with MP-AzeFlu reached this threshold up to 3 days earlier versus FP and up to 5 days earlier versus AZE. Complete or near-complete elimination of symptoms (i.e., reduction in all nasal symptom scores to $\leq 1$ ) was observed in patients treated with MPAzeFlu up to 5 days faster than in those treated with FP $(p=0.033)$ and up to 7 days faster than in those treated with AZE $(p<0.001)$.

Hampel and colleagues [18] investigated the effects of 14 days of treatment with MP-AzeFlu, AZE, or FP for relieving AR symptoms in a randomized, double-blind study of 610 patients aged $\geq 12$ years with a minimum 2 -year history of confirmed allergy to Texas mountain 
cedar pollen. Compared with placebo, all treatments led to significantly improved reflective total nasal symptom score $(p<0.001)$, with MP-AzeFlu being significantly superior to either agent alone $(p<0.01)$. Individual nasal symptoms of nasal congestion, itchy nose, and sneezing were also significantly improved with MP-AzeFlu ( $p \leq$ $0.02)$ [18].

In a post hoc analysis of the study by Hampel and colleagues [18], Meltzer and colleagues [19] assessed the efficacy of MP-AzeFlu versus AZE and FP for controlling nasal and ocular symptoms, irrespective of baseline severity, to understand the clinical relevance. Only MP-AzeFlu was consistently statistically superior to placebo in reducing reflective total nasal symptom score for all patient types, independent of the most severe symptom. More patients treated with MP-AzeFlu achieved a $\geq 30$, $\geq 50, \geq 60, \geq 75$, and $\geq 90 \%$ reflective total nasal symptom score reduction, which occurred days faster than with either active comparator. Approximately $71 \%$ of patients treated with MP-AzeFlu achieved a $\geq 30 \%$ reduction in total nasal symptom score, and half achieved a $\geq 50 \%$ reduction; 1 in 6 achieved complete/near-complete response. MP-AzeFlu significantly decreased nasal congestion, the most bothersome symptom, versus FP and AZE [19]. When comparing this analysis with results from the 3 parallel-group trials using non-commercially available active comparators, the treatment differences were more pronounced, suggesting a formulation effect $[18,19]$.

In a randomized, open-label, active-controlled, parallel-group study, Price and colleagues [21] compared the efficacy of MP-AzeFlu with intranasal FP in 464 patients with persistent rhinitis (chronic allergic or nonallergic rhinitis) over 52 weeks. The study included a 7-days screening period and a 52-weeks treatment period. After 1 month, $73.4 \%$ of patients treated with MP-AzeFlu achieved a $100 \%$ reduction in reflective total nasal symptom score from baseline, a median 8 days faster than the FP group. MP-AzeFlu was more effective than FP, with superior efficacy lasting the whole year, resulting in nearly 30 more symptom-free days (172.8 symptom-free days with MP-AzeFlu vs. 146.9 symptom-free days with FP). Overall, MP-AzeFlu led to a $75 \%$ reduction in symptom score compared with FP [21].

In a pan-European, multicenter, observational study, Klimek and colleagues [26] assessed the effectiveness of MP-AzeFlu in 2,988 patients with AR in routine clinical practice, using a visual analog scale. MP-AzeFlu was associated with an effective and rapid symptom reduction from baseline to $23.4 \pm 20.3 \mathrm{~mm}$ by treatment end, a reduction of $50.4 \pm 26.1 \mathrm{~mm}$, which was significant $(p<$

Literature Review: MP-AzeFlu in Allergic Rhinitis
0.001) from day one and sustained until the last day [26]. Notably, previous studies have defined a $\geq 23 \mathrm{~mm}$ reduction in visual analog scale score as a clinically meaningful difference [33]. Patients treated with MP-AzeFlu rapidly achieved a clinically meaningful response in this study; $26.4 \%$ achieved this reduction at day one, $58.4 \%$ at day 3 , and $79.3 \%$ following 1 week of treatment [26]. Overall, $>93 \%$ of patients treated with MP-AzeFlu reported well or partially controlled symptoms by day 3 . In comparison, the clinically meaningful response in phase 3 clinical studies, defined as a $\geq 30 \%$ reduction in reflective total nasal symptom score [34], was achieved by $71.2 \%$ of patients treated with MP-AzeFlu at day 14 [19].

Kaulsay and colleagues [17] assessed the effectiveness of 6 weeks of MP-AzeFlu treatment for relieving AR symptom severity in 53 Irish patients with persistent $A R$, demonstrating a rapid visual analog scale score reduction from $73.4 \mathrm{~mm}$ at baseline to $31.5 \mathrm{~mm}$ at day $28(p<$ $0.0001)$ and to $28.1 \mathrm{~mm}$ at day $42(p<0.0001)$, which corresponds to a 57 and $62 \%$ change from baseline, respectively. More than half of patients exhibited a clinically significant improvement $(\sim 23 \mathrm{~mm})$ on day 3 and approximately $75 \%$ on the last day of treatment [17]. Using the ARIA-defined cutoff of $50 \mathrm{~mm}$ for controlled symptoms, patients achieved this reduction prior to day 7 , on average. Endoscopy was used to assess edema, discharge, and redness of the nasal mucosa. After treatment with MPAzeFlu, the total endoscopy score significantly decreased, from 7.5 at baseline to 3.5 at day $28(p<0.0001)$. Reductions were observed in the proportion of patients with severe edema ( 53.1 vs. $3.8 \%$ ), thick mucus discharge ( 28.3 vs. $4.8 \%$ ), and severe redness ( 34.9 vs. $0 \%$ ). These results can be compared with findings from a long-term, randomized controlled trial that demonstrated increases in the proportion of patients with no edema ( $20.0 \mathrm{vs} .84 .1 \%)$, no nasal discharge (12.1 vs. $82.6 \%$ ), and no mucosal redness (42.3 vs. $79.3 \%$ ) after 1 year of MP-AzeFlu treatment [22].

Gathering data from both randomized controlled trials and real-world studies is needed to produce a complete evidence base for pharmacologic interventions. This is supported by the inclusion of evidence from randomized controlled trials and real-world data in the development of the recent ARIA guidelines [8]. Because realworld studies have a broad, heterogeneous patient population, the results can be generalized to the entire population of patients with AR, which is not possible with randomized controlled trials [26]. In addition, real-world studies provide results that are more reflective of the level of clinical care delivered in everyday practice. However,

Int Arch Allergy Immunol 2021;182:1026-1035 1029 
because the real-world data presented have no active comparator, the effect size is difficult to interpret against natural improvement of seasonal disease.

In summary, data from randomized controlled trials described above showed that treatment with MP-AzeFlu led to a 44-64\% greater nasal symptom improvement versus comparators (FP and AZE) [20], and a 75\% reduction in symptom score compared with FP in randomized controlled trials [21]. Data from clinical trials further indicate that $71.2 \%$ of patients treated with MP-AzeFlu achieve a clinically meaningful response at day 14 [19], while realworld studies show that $79.3 \%$ of patients achieve a clinically meaningful response following 1 week of treatment [26]. These results support the clinical effects of MP-AzeFlu in the real world.

Olfactory Function and Nasal Hyperreactivity

Patients with AR experience olfactory dysfunction, which appears to be more frequent with increasing AR severity and duration [35], and a positive correlation exists between quality of life and olfaction [36]. Nasal hyperreactivity has been reported by two-thirds of patients with $A R$, and is defined as increased nasal mucosa sensitivity to nonspecific environmental stimuli, leading to nasal symptoms [37]. Antihistamines and topical steroids exert beneficial effects on olfaction; therefore, studies investigating MP-AzeFlu also assessed the impact of treatment.

MP-AzeFlu treatment was associated with significantly improved olfactory threshold, discrimination, and identification scores and symptoms in a multicenter, observational study of 47 patients with persistent AR over time [27]. In addition, a significant interaction among AR severity and olfactory function and visual analog scale was observed. With increasing AR severity, olfactory function decreased; however, greater olfactory improvement was observed in patients diagnosed with more severe AR [27].

In a 4-week, double-blind study of 28 patients with house dust mite AR, MP-AzeFlu significantly reduced nasal hyperreactivity, assessed using a cold dry air provocation protocol, and inflammatory mediators versus placebo [30].

These experimental endpoints for assessing olfactory function and nasal hyperreactivity have been used in previous studies, supporting their use for assessing the effects of treatment. In a prospective clinical trial comparing mometasone furoate and fluticasone furoate, olfactory function was assessed in 24 patients with persistent AR and hyposmia using the extended test battery "Sniffin"
Sticks," and a visual analog scale was used to assess hyposmia and nasal discharge [38]. Similar effects were reported on hyposmia and nasal symptoms between the 2 treatments and the final nasal symptoms score correlated with visual analog scale improvements. The cold dry air provocation protocol was previously validated in a study of 12 patients with AR, 12 patients with idiopathic rhinitis, and 12 healthy patients [39]. This study demonstrated that cold dry air exposure induced nasal obstruction and led to significantly decreased peak nasal inspiratory flow rates, with a high specificity and sensitivity for diagnosis of nasal hyperreactivity of 100 and $66.7 \%$, respectively, highlighting that this method is reliable for the diagnosis of hyperreactivity in patients with rhinitis [39].

\section{Ocular Symptoms}

Ocular symptoms occur in up to $95 \%$ of patients with AR when blinking, squinting, frontal headache, and eyelid dermatitis are considered in combination with itch, redness, watering, and swelling, which make up the total ocular symptom score $[40,41]$. These can substantially impair daily functioning and quality of life [3]. Among patients with ocular symptoms, 53\% recognized they had allergic conjunctivitis, whereas the remaining $40 \%$ exhibited ocular neglect [41]. Intranasal corticosteroids reduce ocular symptoms because of a class effect, whereby intranasal corticosteroids bind to the glucocorticoid receptor leading to increased expression of anti-inflammatory molecules and $\beta$-adrenergic receptors and decreased expression of pro-inflammatory cells and molecules, with further benefits from the addition of an antihistamine [42]. Therefore, the inclusion of an intranasal corticosteroid and an antihistamine in MP-AzeFlu could contribute to decreased ocular symptoms and the use of fewer medications.

In the meta-analysis by Carr and colleagues [20] described previously, MP-AzeFlu led to a significantly reduced average reflective total ocular symptom score from baseline versus FP $(-3.2$ vs. $-2.8 ; p=0.003)$ or placebo $(-3.2$ vs. $-1.8 ; p<0.001)$. This corresponds with differences of 27, 23, and 15\% with MP-AzeFlu, FP, and placebo, respectively [20]. Of note, this effect was also confirmed in the study comparing MP-AzeFlu with commercially available FP and AZE monotherapies [43]. In the study by Hampel and colleagues [18] described above, MP-AzeFlu treatment significantly improved the overall total ocular symptom score compared with placebo and FP $(p<0.01)$. MP-AzeFlu resulted in a $27 \%$ improvement compared with a $21 \%$ improvement with AZE, $18 \%$ for $\mathrm{FP}$, and $11 \%$ for placebo. MP-AzeFlu also improved all 
individual ocular symptoms comparatively $(p<0.05)$ except versus AZE for watery eyes [18].

In the post hoc analysis by Meltzer and colleagues [19], MP-AzeFlu led to a significantly superior change in reflective total ocular symptom score from baseline compared with both FP and AZE, with a relative difference of $63 \%$ versus $\mathrm{FP}$ and $42 \%$ versus AZE, despite moderate-tosevere ocular symptoms at baseline. Considering reflective total ocular symptom score plus reflective total nasal symptom score, patients treated with MP-AzeFlu exhibited a greater improvement, of 52 and $56 \%$, versus patients treated with FP $(p=0.0013)$ or AZE $(p<0.0004)$, respectively [19]. With MP-AzeFlu, significant improvements in ocular itching and redness were reported versus FP, AZE, and placebo, and in ocular watering versus FP and placebo [19]. The treatment effects in this study were consistently higher than in the meta-analysis by Carr and colleagues $[19,20]$, and may be attributable to formulation and device effects.

\section{Time to Onset of Effect}

Other treatments, including single-agent FP and AZE and the combination of an intranasal corticosteroid and oral antihistamine, do not provide the same level of efficacy as MP-AzeFlu for rapid and sustained relief of all AR symptoms. A previous study demonstrated an onset of action for the combination of intranasal FP and oral loratadine of 150 versus $5 \mathrm{~min}$ for MP-AzeFlu - a difference of nearly two and a half hours [6].

Environmental exposure chambers offer advantages for assessing onset of action that can be demonstrated in minutes [8]. The chamber allows for consistent allergen exposure, whereas in a typical phase 3 study, the first dose and early assessment are conducted under an indoor, low-pollen exposure condition [8]. In a singlecenter, randomized, placebo-controlled crossover trial of 78 asymptomatic patients, AR symptoms were induced by ragweed pollen challenge in an environmental exposure chamber [29]. Bousquet and colleagues [29] reported a clinically relevant (defined as a $30-50 \%$ symptom reduction) and significant effect of MP-AzeFlu versus placebo at 5 min until assessment end ( $4 \mathrm{~h}$; $p \leq 0.04)$. Meltzer and colleagues [44] reported a similar rapid onset of action of MP-AzeFlu in a trial setting, with significantly greater total nasal symptom score improvement versus placebo after $30 \mathrm{~min}$ and at all subsequent evaluations.

In a single-center, single-dose, randomized, doubleblind trial, 425 patients were exposed to ragweed in an environmental exposure chamber [45]. Patients treated

Literature Review: MP-AzeFlu in Allergic Rhinitis with mometasone experienced significant symptom reduction compared with placebo at $150 \mathrm{~min}$ postdose [45].

A randomized study of 450 adults exposed to ragweed pollen in an environmental exposure chamber showed that treatment with AZE nasal spray led to a significant $45 \%$ reduction of total nasal symptom score from baseline, starting $15 \mathrm{~min}$ after administration and lasting throughout the 8-h allergen exposure [46].

\section{Effects on Quality of Life}

AR can profoundly impact quality of life and contribute to a greater impairment of work productivity than type 2 diabetes and hypertension $[1,4]$. Therefore, studies were designed to investigate associations between patient-reported changes in quality of life and MP-AzeFlu treatment.

MP-AzeFlu significantly improved the overall Rhinoconjunctivitis Quality of Life Questionnaire score versus placebo among 779 patients with moderate-to-severe seasonal AR [44]. In the analysis by Carr and colleagues [20], 14 days of treatment with MP-AzeFlu, FP, or AZE led to significantly improved quality of life. In the Hampel and colleagues [18] study previously described, MPAzeFlu treatment significantly improved nasal congestion; runny nose; itchy nose; sneezing; and itchy, watery, and red eyes, leading to significant improvements in the overall score and each domain of the Rhinoconjunctivitis Quality of Life Questionnaire compared with placebo $(p<0.001)$ and AZE $(p=0.005)$. In a noninterventional study of patients with moderate-to-severe AR treated with MP-AzeFlu for $\sim 14$ days, patients reported improvements in mean visual analog scale scores for impairment in sleep quality, work or school daily activity, social activity, and outdoor activity (Van Weissenbruch, et al. Unpublished data). Of importance, these results were similar across all populations, regardless of Immunoglobulin E-mediated disease phenotype, comorbidity, sex, and age. Finally, in noninterventional studies of patients with AR conducted in Ireland, increased rates of patients with very good or good sleep quality from baseline (25\%) through day 28 (78.4\%) were seen with MPAzeFlu treatment [17].

These experimental endpoints for assessing quality of life and sleep quality have been used in previous studies of AZE and FP, supporting their use for assessing the effects of treatment. Results from a 14-days study of patients with moderate-to-severe seasonal AR demonstrated significant improvements from baseline in the Rhinoconjunctivitis Quality of Life Questionnaire score among 
patients treated with AZE compared with placebo [47]. Among 24 subjects treated with AZE or placebo for 8 weeks, AZE-treated patients reported significant improvements in sleep after treatment that were superior to those reported by the placebo-treated group [48]. Among patients aged $\geq 12$ years with seasonal AR, once-daily treatment for 2 weeks with fluticasone furoate nasal spray led to clinically significantly improved Rhinoconjunctivitis Quality of Life Questionnaire score [49]. Of note, this clinical difference was achieved in the individual domain of sleep problems [49]. In a study of 19 patients aged $\geq 18$ years with physician-diagnosed asthma and mild AR, 6 weeks of treatment with fluticasone propionate led to a significant increase in the Rhinoconjunctivitis Quality of Life Questionnaire index compared with placebo; however, no significant changes in sleep from baseline were observed [50]. Results from a randomized study of patients with seasonal AR demonstrated that fluticasone furoate nasal spray was more effective than placebo in terms of nighttime sleep disturbances caused by seasonal AR symptoms [51].

Overall, the use of MP-AzeFlu improves the severity of AR symptoms and patient quality of life by reducing the impact on sleep and daily activities, regardless of phenotype or comorbidity. With its rapid time to onset, MPAzeFlu quickly improves symptoms and lessens the profound impact of AR on patient quality of life.

\section{Safety}

When developing combination therapies, potential drug-drug interactions between active components or formulation-based alterations of bioavailability must be investigated [52]. The initial efficacy of MP-AzeFlu warranted assessment of its safety and tolerability. No evidence of pharmacokinetic interactions between AZE and FP in MP-AzeFlu has been reported [12].

In randomized, crossover studies of healthy subjects, no interactions were found between AZE and FP with MP-AzeFlu [16]. Furthermore, AZE bioavailability was similar between MP-AzeFlu and the MP-AzeFlu-based product containing only AZE. FP exposure was higher for MP-AzeFlu-based products versus FP alone; however, FP concentrations were very low among all products, suggesting no clinically meaningful differences in systemic safety [16].

In a 1-year randomized, open-label, active-controlled, parallel-group study of 612 patients with chronic AR or nonallergic rhinitis, patients received one spray per nostril of MP-AzeFlu twice daily or 2 sprays per nostril of FP once daily [22]. The incidences of treatment-related ad- verse events were low with both MP-AzeFlu (9.4\%) and FP (11.1\%), with no evidence of late-occurring adverse events. No findings would preclude the long-term (52-weeks) use of MP-AzeFlu in the treatment of AR. The most common treatment-related adverse events were headache (4.3\%) with FP and dysgeusia (2.5\%) with MPAzeFlu. No appreciable reduction in serum cortisol was seen from baseline after 12 months of continuous treatment with either MP-AzeFlu $(-0.08 \pm 5.5 \mathrm{mcg} / \mathrm{dL})$ or FP $(-1.04 \pm 5.0 \mathrm{mcg} / \mathrm{dL})[22]$.

In the real-life, pan-European study by Klimek and colleagues [26], treatment with MP-AzeFlu was well tolerated, with $<1 \%$ of nearly 3,000 treated patients reporting adverse events. The most commonly reported adverse events were dysgeusia $(n=4)$, nausea $(n=3)$, sneezing $(n=3)$, and nasal discomfort $(n=3)$. Rhinorrhea, application-site pain, and epistaxis were also reported by 2 patients each [26]. In Kaulsay and colleagues' [17] noninterventional study of 53 patients, 1 patient reported fatigue and 1 patient reported sedation.

\section{Special Populations}

In a multicenter, prospective, noninterventional study of patients with moderate-to-severe seasonal or perennial AR treated with MP-AzeFlu for $\sim 14$ days, mean visual analog scale scores similarly decreased for impairment of sleep quality among participants aged 12-17 years, 18-65 years, and $>65$ years. (Van Weissenbruch, et al. Unpublished data). Similar results were reported across age groups for visual analog score improvement in impairment of daily work or school, social, and outdoor activities (Van Weissenbruch, et al. Unpublished data).

In an open-label trial of 405 children aged 4 to $<12$ years with a history of AR randomized to receive MPAzeFlu or FP for 3 months, MP-AzeFlu led to a significantly greater reduction in total symptom score [24]. This difference was noted from the first assessment and was sustained for 90 days. In the first month, $80 \%$ of children treated with MP-AzeFlu reported no symptoms or mild symptom severity, which was achieved up to 16 days quicker than with FP. More children who received MPAzeFlu experienced mild or no symptoms throughout the study period versus FP (73.4 vs. 66.0\%) [24].

In a 14-days, randomized study of 304 children aged 6-11 years with moderate-to-severe AR, a significant improvement in Paedeatric Rhinoconjunctivitis Quality of Life Questionnaire overall score was reported with MPAzeFlu versus placebo [25]. When children mostly rated their own symptoms, MP-AzeFlu led to significantly im- 
proved relief versus placebo for reflective total nasal symptom score, reflective total ocular symptom score, and individual nasal and ocular symptoms [25].

In a 3-month, open-label study of children aged 4-11 years with AR, the incidence of treatment-related adverse events was low with MP-AzeFlu or FP alone (16 vs. 12\%) [28]. The most frequent adverse event was epistaxis, which was reported in $9 \%$ of patients in both groups. No mucosal ulceration or nasal septal perforation was reported and there were no unusual changes in vital signs or laboratory parameters, demonstrating the safety and tolerability of MP-AzeFlu in children [28].

\section{Competitor Landscape}

In a phase 3 , double-blind, randomized study of 1,180 patients, 14 days of GSP301, an investigational, fixeddose combination of olopatadine hydrochloride and mometasone furoate delivered in a nasal spray device, significantly improved the reflective total nasal symptom score versus placebo and olopatadine alone, but not mometasone furoate alone; the instantaneous total nasal symptom score was significantly improved versus placebo and both monotherapies [53]. GSP301 significantly improved individual nasal symptoms, reflective total ocular symptom score, instantaneous total ocular symptom score, and quality of life versus placebo, with an onset of action within $15 \mathrm{~min}$. Overall, 12.9\% of GSP301-treated patients reported treatment-emergent adverse events, including dysgeusia (3.3\%), which was similar to olopatadine alone (3.0\%) [53].

Although the efficacy and safety of MP-AzeFlu and GSP301 are similar, the dosing of GSP301 is 2 sprays per nostril twice daily, whereas MP-AzeFlu requires only one spray per nostril twice daily $[12,53]$, which may be associated with increased adherence. Compared with mometasone, GSP301 was not significantly superior, whereas MP-AzeFlu treatment led to significant symptom improvements, suggesting GSP301 may be less effective than MP-AzeFlu.

In a randomized, double-blind, double-dummy study of 180 participants, treatment with twice-daily GSP301 led to a significant difference in instantaneous total nasal symptom score change from baseline after $10 \mathrm{~min}$ versus placebo [31]. No significant difference in instantaneous total nasal symptom score change was seen with MP-AzeFlu. However, MP-AzeFlu was administered with one spray of placebo in this study, which reduced its effectiveness and impacted time to onset because of a washout effect. Additional direct comparisons are needed to determine any true differences.

\section{Conclusion}

This body of literature suggests that treatment options, such as single-agent FP or AZE and the combination of an intranasal corticosteroid and an oral antihistamine do not provide the same level of efficacy as MP-AzeFlu in terms of rapid and sustained relief of the entire AR symptom complex. Taken together, these studies further demonstrate that MP-AzeFlu treatment also improves quality of life. MP-AzeFlu is the only currently available combination that satisfies patient needs and expectations, such as prompt relief, effects on both nasal and ocular symptoms in all patient types, and preference for an intranasal, combination treatment. The use of MP-AzeFlu as firstline therapy for AR is supported by evidence-based medical guidelines. Following the example of combination therapy in asthma control [54], first-line combination therapy for AR may improve symptom control, patient adherence, disease management, and overall quality of life.

\section{Acknowledgements}

Technical, editorial, and medical writing assistance were provided under the direction of the authors by Erin Burns, $\mathrm{PhD}$, and Strategix, an affiliate of The Lynx Group, LLC. Funding for this support was provided by Mylan Inc.

\section{Conflict of Interest Statement}

Ludger Klimek reports financial grants from Allergopharma, ALK Abello, Allergy Therapeut, Stallergenes, Quintiles, HAL Allergie, LETI Pharma, Sanofi, AstraZeneca, GSK, ASIT biotech \& Lofarma. He has also been a paid consultant for Allergopharma, MEDA/Mylan, HAL Allergie, ALK Abello \& LETI Pharma; William Berger reports being a paid consultant for ALK Regeneron/ Sanofi Genzyme and has been a paid speaker for AstraZeneca, TEVA, Optinose; Jean Bousquet has nothing to report; Paul K. Keith has received financial grants from Astra, CSL, Behring, Genentech, Shire (Takeda). He has also been a paid consultant/speaker for ALK, Astra, CSL, Behring, GSK, Kaleo, Merck, Mylan, Novartis, Pediapharm, Sanofi, Shire (Takeda); Peter Smith has had an Investigator initiated grant from Mylan and is on the Advisory Board for Nestle, Seqaris, Novartis, Sonofi and Mylan; Dirceu Sole has nothing to report; Glenis Scadding has had research funding from GSK, fees for consultancy from ALK, Mylan, Sanofi-Regeneron, GSK and speaker fees from Mylan, ALK, Bayer, GSK; HansChristian Kuhl is an employee of Mylan, Inc.; Duc Tung Nguyen is an employee of MEDA GmbH \& Co. KG (A Mylan Company); Ferdinand Kopietz is an employee of Mylan, Inc.; Arkady Koltun has nothing to report. 


\section{Funding Sources}

This study was supported by Meda Pharma GmbH \& Co. KG (a Mylan Company), Bad Homburg, Germany.

\section{Author Contributions}

All authors made substantial contributions to the conception or design of the manuscript, or the acquisition, analysis, or interpretation of data for the manuscript, and all authors were involved in drafting the manuscript or revising it critically for important intellectual content. The authors were fully responsible for all content and editorial decisions, and received no financial support or other form of compensation related to the development of this manuscript. All authors had final approval of the manuscript and are accountable for all aspects of the work in ensuring the accuracy and integrity of this manuscript.

\section{Availability of Data and Material}

The data that support the findings of this study are available from the corresponding author upon reasonable request.

\section{References}

1 Bousquet J, Khaltaev N, Cruz AA, Denburg J, Fokkens WJ, Togias A, et al. Allergic rhinitis and its impact on asthma (ARIA) 2008 update (in collaboration with the World Health Organization, GA(2)LEN and AllerGen). Allergy. 2008;63(Suppl 86):8-160.

2 Canonica GW, Mullol J, Pradalier A, Didier A. Patient perceptions of allergic rhinitis and quality of life: findings from a survey conducted in Europe and the United States. World Allergy Organ J. 2008;1(9):138-44.

3 Canonica GW, Bousquet J, Mullol J, Scadding GK, Virchow JC. A survey of the burden of allergic rhinitis in Europe. Allergy. 2007; 62(Suppl 85):17-25.

4 de la Hoz Caballer B, Rodríguez M, Fraj J, Cerecedo I, Antolín-Amérigo D, Colás C. Allergic rhinitis and its impact on work productivity in primary care practice and a comparison with other common diseases: the cross-sectional study to evAluate work productivity in allergic rhinitis compared with other common dIseases (CAPRI) study. Am J Rhinol Allergy. 2012;26(5):390-4.

5 Hellings PW, Dobbels F, Denhaerynck K, Piessens M, Ceuppens JL, De Geest S. Explorative study on patient's perceived knowledge level, expectations, preferences and fear of side effects for treatment for allergic rhinitis. Clin Transl Allergy. 2012;2(1):9.

6 Bachert C, Bousquet J, Hellings P. Rapid onset of action and reduced nasal hyperreactivity: new targets in allergic rhinitis management. Clin Transl Allergy. 2018;8:25.

7 Bousquet J, Schünemann HJ, Hellings PW, Arnavielhe S, Bachert C, Bedbrook A, et al. MACVIA clinical decision algorithm in adolescents and adults with allergic rhinitis. J Allergy Clin Immunol. 2016;138(2):367e2.

8 Bousquet J, Schünemann HJ, Togias A, Bachert C, Erhola M, Hellings PW, et al. Next-generation allergic rhinitis and its impact on asthma (ARIA) guidelines for allergic rhinitis based on grading of recommendations assessment, development and evaluation (GRADE) and real-world evidence. J Allergy Clin Immunol. 2020;145(1):70-e3.
9 Tan R, Cvetkovski B, Kritikos V, Price D, Yan $\mathrm{K}$, Smith $\mathrm{P}$, et al. Identifying the hidden burden of allergic rhinitis (AR) in community pharmacy: a global phenomenon. Asthma Res Pract. 2017;3:8.

10 Wahid NWB, Shermetaro C. Rhinitis medicamentosa. Treasure Island, FL: StatPearls; 2019.

11 Jáuregui I, Mullol J, Dávila I, Ferrer M, Bartra J, del Cuvillo A, et al. Allergic rhinitis and school performance. J Investig Allergol Clin Immunol. 2009;19(Suppl 1):32-9.

12 EMC [Internet]. Dymista 137 micrograms/50 micrograms per actuation nasal spray. Summary of product characteristics [cited 2020 Sep 29]. 2019. Available from: www.medicines.org.uk/emc/product/9450/smpc.

13 Wallace DV, Dykewicz MS, Oppenheimer J, Portnoy JM, Lang DM. Pharmacologic treatment of seasonal allergic rhinitis: synopsis of guidance from the 2017 joint task force on practice parameters. Ann Intern Med. 2017; 167(12):876-81.

14 Dykewicz MS, Wallace DV, Baroody F, Bernstein J, Craig T, Finegold I, et al. Treatment of seasonal allergic rhinitis: an evidence-based focused 2017 guideline update. Ann Allergy Asthma Immunol. 2017;119(6):489-511.

15 Meltzer EO, Bachert C, Mayer MJ, Kopietz F, Koltun A, Maus J, et al. Deposition characteristics of a novel intranasal formulation of azelastine hydrochloride plus fluticasone propionate in an anatomic model of the human nasal cavity. Allergy Asthma Proc. 2020; 41(4):265-70

16 Derendorf H, Munzel U, Petzold U, Maus J, Mascher H, Hermann R, et al. Bioavailability and disposition of azelastine and fluticasone propionate when delivered by MP29-02, a novel aqueous nasal spray. Br J Clin Pharmacol. 2012;74(1):125-33.

17 Kaulsay R, Nguyen DT, Kuhl HC. Real-life effectiveness of MP-AzeFlu in Irish patients with persistent allergic rhinitis, assessed by visual analogue scale and endoscopy. Immun Inflamm Dis. 2018;6(4):456-64.
18 Hampel FC, Ratner PH, Van Bavel J, Amar NJ, Daftary P, Wheeler W, et al. Doubleblind, placebo-controlled study of azelastine and fluticasone in a single nasal spray delivery device. Ann Allergy Asthma Immunol. 2010; 105(2):168-73.

19 Meltzer E, Ratner P, Bachert C, Carr W, Berger W, Canonica GW, et al. Clinically relevant effect of a new intranasal therapy (MP2900302) in allergic rhinitis assessed by responder analysis. Int Arch Allergy Immunol. 2013;161(4):369-77.

20 Carr W, Bernstein J, Lieberman P, Meltzer E, Bachert C, Price D, et al. A novel intranasal therapy of azelastine with fluticasone for the treatment of allergic rhinitis. J Allergy Clin Immunol. 2012;129(5):1282-e10.

21 Price D, Shah S, Bhatia S, Bachert C, Berger W, Bousquet J, et al. A new therapy (MP2902) is effective for the long-term treatment of chronic rhinitis. J Investig Allergol Clin Immunol. 2013;23(7):495-503.

22 Berger WE, Shah S, Lieberman P, Hadley J, Price D, Munzel U, et al. Long-term, randomized safety study of MP29-02 (a novel intranasal formulation of azelastine hydrochloride and fluticasone propionate in an advanced delivery system) in subjects with chronic rhinitis. J Allergy Clin Immunol Pract. 2014; 2(2):179-85.

23 Klimek L, Bachert C, Mösges R, Munzel U, Price D, Virchow JC, et al. Effectiveness of MP29-02 for the treatment of allergic rhinitis in real-life: results from a noninterventional study. Allergy Asthma Proc. 2015;36(1):40-7.

24 Berger W, Bousquet J, Fox AT, Just J, Muraro A, Nieto A, et al. MP-AzeFlu is more effective than fluticasone propionate for the treatment of allergic rhinitis in children. Allergy. 2016; 71(8):1219-22.

25 Berger W, Meltzer EO, Amar N, Fox AT, Just $\mathrm{J}$, Muraro A, et al. Efficacy of MP-AzeFlu in children with seasonal allergic rhinitis: importance of paediatric symptom assessment. Pediatr Allergy Immunol. 2016;27(2):12633. 
26 Klimek L, Bachert C, Stjärne P, Dollner R, Larsen $\mathrm{P}$, Haahr P, et al. MP-AzeFlu provides rapid and effective allergic rhinitis control in real life: a pan-European study. Allergy Asthma Proc. 2016;37(5):376-86.

27 Klimek L, Poletti SC, Sperl A, Spielhaupter M, Bardenhewer C, Mullol J, et al. Olfaction in patients with allergic rhinitis: an indicator of successful MP-AzeFlu therapy. Int Forum Allergy Rhinol. 2017;7(3):287-92.

28 Berger W, Sher E, Gawchik S, Fineman S. Safety of a novel intranasal formulation of azelastine hydrochloride and fluticasone propionate in children: a randomized clinical trial. Allergy Asthma Proc. 2018;39(2):110-6.

29 Bousquet J, Meltzer EO, Couroux P, Koltun A, Kopietz F, Munzel U, et al. Onset of action of the fixed combination intranasal azelastine-fluticasone propionate in an allergen exposure chamber. J Allergy Clin Immunol Pract. 2018 Sep-Oct;6(5):1726-e6.

30 Kortekaas Krohn I, Callebaut I, Alpizar YA, Steelant B, Van Gerven L, Skov PS, et al. MP29-02 reduces nasal hyperreactivity and nasal mediators in patients with house dust mite-allergic rhinitis. Allergy. 2018;73(5): 1084-93.

31 Patel P, Salapatek AM, Tantry SK. Effect of olopatadine-mometasone combination nasal spray on seasonal allergic rhinitis symptoms in an environmental exposure chamber study. Ann Allergy Asthma Immunol. 2019;122(2): $160-\mathrm{e} 1$.

32 Debbaneh PM, Bareiss AK, Wise SK, McCoul ED. Intranasal azelastine and fluticasone as combination therapy for allergic rhinitis: systematic review and meta-analysis. Otolaryngol Head Neck Surg. 2019;161(3):412-8.

33 Demoly P, Bousquet PJ, Mesbah K, Bousquet $\mathrm{J}$, Devillier P. Visual analogue scale in patients treated for allergic rhinitis: an observational prospective study in primary care: asthma and rhinitis. Clin Exp Allergy. 2013;43(8): 881-8.

34 Meltzer EO, Wallace D, Dykewicz M, Shneyer L. Minimal clinically important difference (MCID) in allergic rhinitis: agency for healthcare research and quality or anchor-based thresholds? J Allergy Clin Immunol Pract. 2016;4(4):682-e6.

35 Rhee CS, Wee JH, Ahn JC, Lee WH, Tan KL, Ahn S, et al. Prevalence, risk factors and comorbidities of allergic rhinitis in South Korea: the Fifth Korea National Health and Nutrition Examination Survey. Am J Rhinol Allergy. 2014;28(2):e107-14.

36 Katotomichelakis M, Simopoulos E, Tripsianis G, Prokopakis E, Danielides G, Velegrakis $S G$, et al. Improvement of olfactory function for quality of life recovery. Laryngoscope. 2013;123(11):E10-6.

37 Segboer CL, Holland CT, Reinartz SM, Terreehorst I, Gevorgyan A, Hellings PW, et al. Nasal hyper-reactivity is a common feature in both allergic and nonallergic rhinitis. Allergy. 2013;68(11):1427-34.

38 Catana IV, Chirila M, Negoias S, Bologa R, Cosgarea M. Effects of corticosteroids on hyposmia in persistent allergic rhinitis. Clujul Med. 2013;86(2):117-20.

39 Van Gerven L, Boeckxstaens G, Jorissen M, Fokkens W, Hellings PW. Short-time cold dry air exposure: a useful diagnostic tool for nasal hyperresponsiveness. Laryngoscope. 2012; 122(12):2615-20.

40 Origlieri C, Bielory L. Intranasal corticosteroids and allergic rhinoconjunctivitis. Curr Opin Allergy Clin Immunol. 2008;8(5):4506.

41 Williams DC, Edney G, Maiden B, Smith PK. Recognition of allergic conjunctivitis in patients with allergic rhinitis. World Allergy Organ J. 2013;6(1):4.

42 Seresirikachorn K, Chitsuthipakorn W, Kanjanawasee D, Khattiyawittayakun L, Snidvongs $\mathrm{K}$. Effects of $\mathrm{H} 1$ antihistamine addition to intranasal corticosteroid for allergic rhinitis: a systematic review and meta-analysis. Int Forum Allergy Rhinol. 2018;8(10):1083-92.

43 Engel J, Emons G, Pinski J, Schally AV. AEZS108: a targeted cytotoxic analog of LHRH for the treatment of cancers positive for LHRH receptors. Expert Opin Investig Drugs. 2012; 21(6):891-9.

44 Meltzer EO, LaForce C, Ratner P, Price D, Ginsberg D, Carr W. MP29-02 (a novel intranasal formulation of azelastine hydrochloride and fluticasone propionate) in the treatment of seasonal allergic rhinitis: a randomized, double-blind, placebo-controlled trial of efficacy and safety. Allergy Asthma Proc. 2012; 33(4):324-32.

45 Patel D, Garadi R, Brubaker M, Conroy JP, Kaji Y, Crenshaw K, et al. Onset and duration of action of nasal sprays in seasonal allergic rhinitis patients: olopatadine hydrochloride versus mometasone furoate monohydrate. Allergy Asthma Proc. 2007;28(5):592-9.

46 Patel P, D'Andrea C, Sacks HJ. Onset of action of azelastine nasal spray compared with mometasone nasal spray and placebo in subjects with seasonal allergic rhinitis evaluated in an environmental exposure chamber. Am J Rhinol. 2007;21(4):499-503.

47 Howland WC, Amar NJ, Wheeler W, Sacks H. Efficacy and safety of azelastine $0.15 \%$ nasal spray administered once daily in patients with allergy to Texas mountain cedar pollen. Int Forum Allergy Rhinol. 2011;1(4):275-9.

48 Golden S, Teets SJ, Lehman EB, Mauger EA, Chinchilli V, Berlin JM, et al. Effect of topical nasal azelastine on the symptoms of rhinitis, sleep, and daytime somnolence in perennial allergic rhinitis. Ann Allergy Asthma Immunol. 2000;85(1):53-7.

49 Fokkens WJ, Jogi R, Reinartz S, Sidorenko I, Sitkauskiene B, van Oene C, et al. Once daily fluticasone furoate nasal spray is effective in seasonal allergic rhinitis caused by grass pollen. Allergy. 2007;62(9):1078-84.

50 Rimmer J, Greenwood A, Bartlett D, Hellgren J. Nasal steroids improve regulation of nasal patency in asthma and mild rhinitis: a randomised, cross-over trial. Eur Arch Otorhinolaryngol. 2012;269(4):1133-8.

51 Andrews CP, Martin BG, Jacobs RL, Mohar DE, Diaz JD, Amar NJ, et al. Fluticasone furoate nasal spray is more effective than fexofenadine for nighttime symptoms of seasonal allergy. Allergy Asthma Proc. 2009;30(2): $128-38$

52 Guideline on clinical development of fixed combination medicinal products. Committee for Human Medicinal Products (CHMP); 2017. Available from: www.ema.europa.eu/ en/documents/scientific-guideline/guideline-clinical-development-fixed-combination-medicinal-products-revision-2_en.pdf Accessed 2020 Feb 4.

53 Hampel FC, Pedinoff AJ, Jacobs RL, Caracta CF, Tantry SK. Olopatadine-mometasone combination nasal spray: evaluation of efficacy and safety in patients with seasonal allergic rhinitis. Allergy Asthma Proc. 2019; 40(4):261-72.

54 Papi A. A new combination therapy for asthma: bridging the gap between effectiveness in trials and clinical practice? Respir Med. 2012; 106(Suppl 1):S1-3. 